\title{
Study on Mechanical Properties of Nanocrystal Surface Layer of an Aluminum Alloy
}

\author{
You-Li Ma, Ji-Jia Xie, You-Shi Hong
}

State Key Laboratory of Nonlinear Mechanics, Institute of Mechanics, Chinese Academy of Sciences, Beijing 100080,China,Email: hongys@imech.ac.cn

\section{Zhong-Min Deng}

School of Space Technology, Beijing University of Aeronautics and Astronautics, Beijing 100083, China

\begin{abstract}
The nanocrystal surface layer of an aluminum alloy induced by High Speed Shot Peening (HSSP) was investigated in this paper. The results of nanoindentation experiment show that the elastic modulus and the hardness of nanocrystal surface layer increased, by $8 \%$ and $20 \%$, respectively. The elastic modulus and the hardness appear to be independent of the distance from nanocrystalized surface and the process time.
\end{abstract}

Keywords: Nanocrystal surface layer, mechanical property, aluminum alloy, nano-hardness, elastic modulus

\section{Introduction}

Nanostructure materials have attracted a growing scientific interest of research. These materials have encouraged expectations of their unusual and extraordinary changes in both mechanical and physical properties that are fundamentally different from, and often far superior to, those of their conventional coarsegrained polycrystalline counterparts.

Severe Plastic Deformation (SPD) is an effective method for making the nanostructure materials by applying intense plastic strain into metals and alloys ${ }^{[1-3]}$. SPD method has two significant advantages over other techniques such as inert gas condensation and high-energy ball milling. First, it is possible to produce large bulk samples. Secondly, these samples are free from any residual porosity and contamination. Several techniques are now available for producing high plastic strain of the order of several hundred percent, including equal channel angular pressure (ECAP), severe plastic torsion staining (SPTS), multiple forging and repetitive corrugation strengthening (RCS).

An SPD based mechanical treatment for surface nanocrystallization (SNC), namely ultrasonic shot peening (USSP), is recently proposed $^{[4]}$. It has been demonstrated that during the process of USSP, the ultra-fine microstructure is produced through introducing intense strain and high strain rates into the surface layer. The strain presents gradient distribution in the surface layer and changes from the maximum at the surface to zero far underneath the substrate. In the present study, the variation of hardness and elastic modulus of an aluminum alloy nanocrystallized surface layers by High Speed Shot Peening (HSSP) are investigated with nano-indentor equipment, and the effects of grain size and process time by High Speed Shot Peening on hardness and elastic modulus are also discussed. 


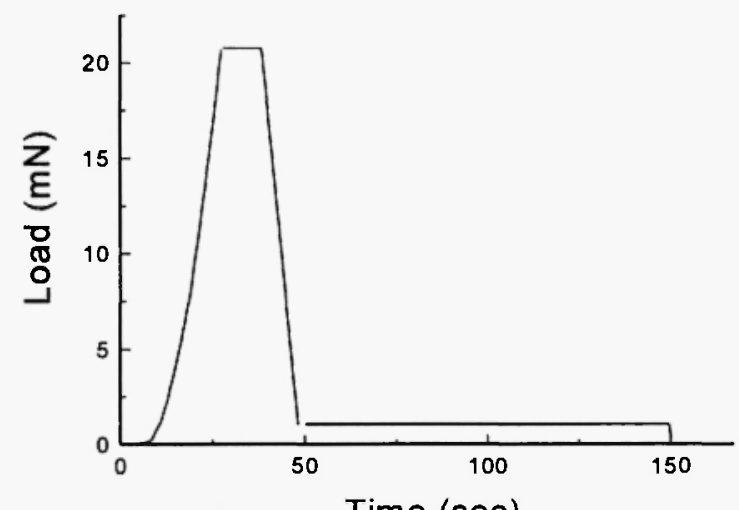

a) before Time (sec)

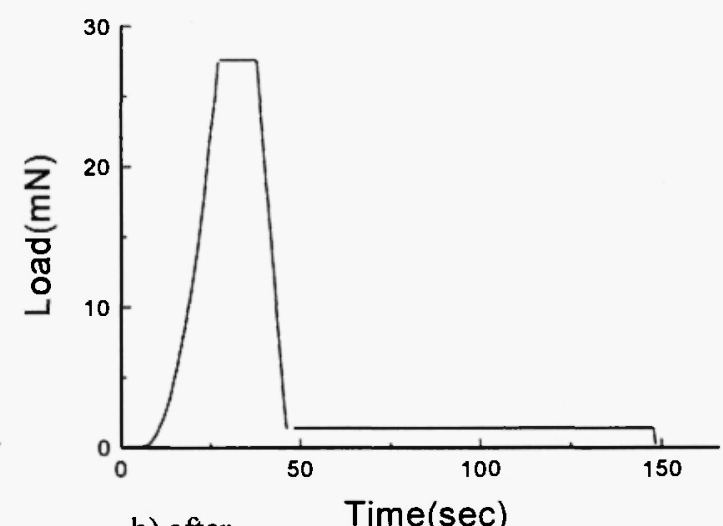

b) after

Fig. 1 Time-load curve applied to the sample before and after nanocrystalization

\section{Experimental Material and Method}

The experimental material is an aluminum alloy with a chemical composition (wt \%) of $2.5 \mathrm{Mg}, 5.6 \mathrm{Zn}, 2.2 \mathrm{Cu}$ and balance $\mathrm{Al}$. $\mathrm{A}$ commercial available plate was cut into pieces with $100 \times 100 \times 10 \mathrm{~mm}^{3}$ in dimension. Before the High Speed Shot Peening, specimens were polished to gain a smooth surface ${ }^{[5]}$.

In brief, the High Speed Shot Peening technique can be described as follows: A highenergy generator vibrates a reflecting chamber with a frequency about $45 \mathrm{~Hz}$, in which there are many stainless steel shots with $7.5 \mathrm{~mm}$ in diameter. The shots resonate and peen the surface of specimen in different directions with high-speed ${ }^{[6,7]}$. As a result, the severe plastic deformation was induced in the surface layer of specimen by the impact load. The strain changes from the maximum value to zero with the distance from the specimen surface to the substrate. In this investigation, the High Speed Shot Peening process was done in vacuum at room temperature for $5,10,15$ and 20 minutes, respectively.

After the High Speed Shot Peening, TEM samples were made for the measurement of the grain size in different positions from nanocrystalized surface. The metallographic samples were also prepared.

Nano-hardness measurement was carried out on Nano Indentor II in the mode control of tip displacement. The maximum displace- ment of the tip is $720 \mathrm{~nm}$.

Figure 1 shows the load-time curves applied to the specimens before and after High Speed Shot Peening. During the test, the indentor moved to the maximum displacement with the velocity of $36 \mathrm{~nm}$ per second. The indentor kept the load for 10 seconds and then it unloaded with the same velocity with the loading course.

The indentation experiment was performed on the sections of every specimen along the line from nanocrystalized surface to the substrate. The distance between any two neighboring indentations is about $12 \mu \mathrm{m}$. Figure 2 shows an example of indentations on a specimen.

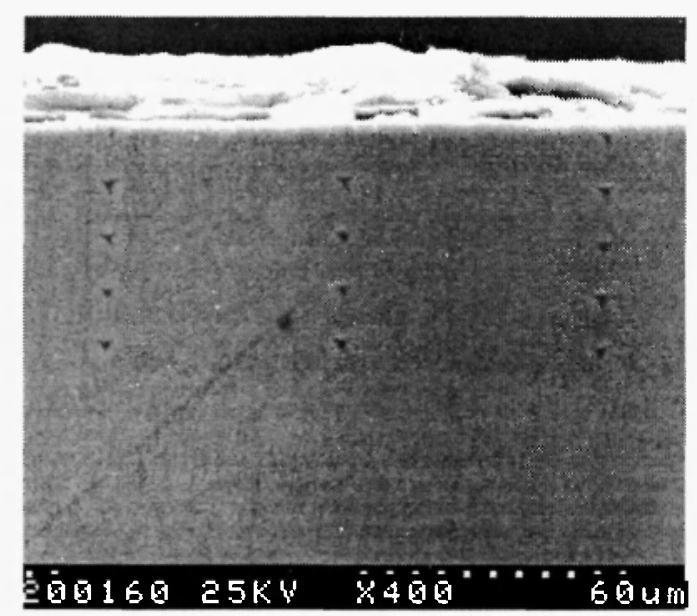

Fig. 2 Indentations on the section of a specimen 


\section{Results and Discussion}

Figure 3 is a metallograph of one specimen after HSSP. It is seen that a thin layer of fine grain size formed at the surface of the specimen. The relationship between the distance from surface and the grain size is shown in Fig 4. It was measured by image analysis on TEM photographs, which were well prepared at the

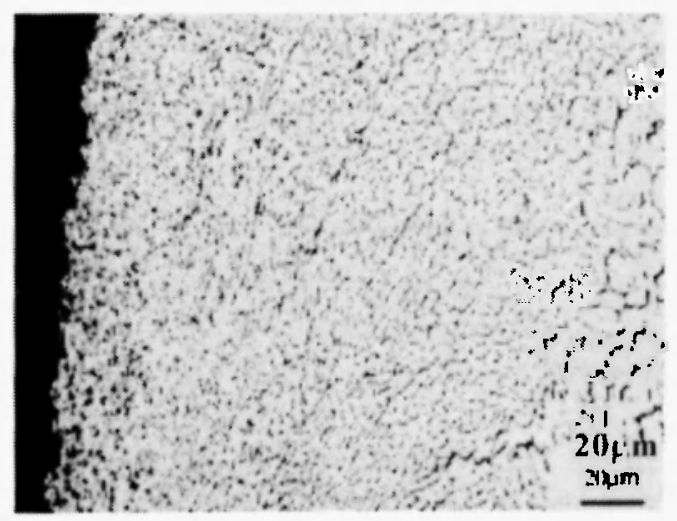

Fig.3 Metallograph of the section of one specimen after High Speed Shot Peening

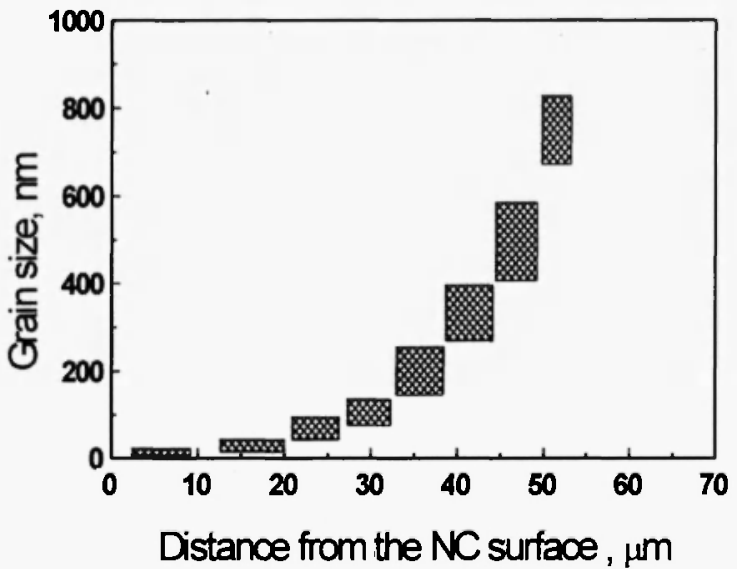

Fig.4 Statistic grain size versus Distance from the NC surface

different positions from nanocrystalized surface. One of such TEM photographs is showed in Fig 5.

Figure 6 shows the relation of the values for elastic modulus and nanohardness versus the distance from the NC structure surface. It is seen that, after High Speed Shot Peening, the elastic modulus and the nanohardness are both

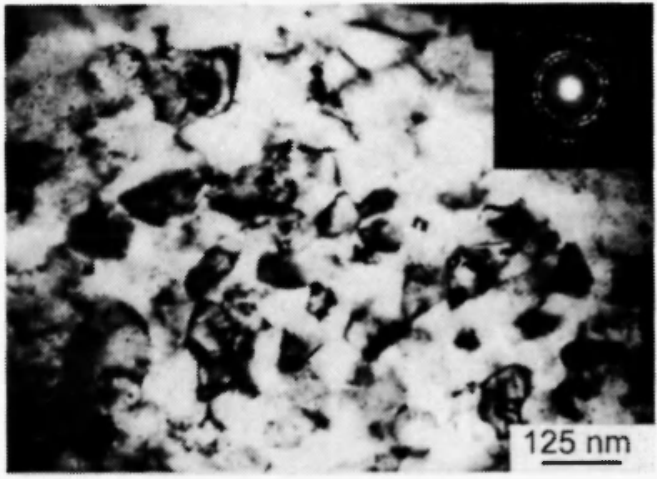

Fig.5 TEM photograph of a specimen after High Speed Shot Peening

increased. The elastic modulus increased by $8 \%$ and the nanohardness increased by $20 \%$ compared to the original state of material and the substrate, which is far from the surface. But it seems that the elastic modulus and the nanohardness change little with the distance from nanocrystalized surface in the domain near the surface. In other words, the elastic modulus and the nanohardness are independent of grain size within such a thin layer under the surface. When the process time of High Speed Shot Peening is longer than 5 minutes, the elastic modulus and the nanohardness do not change much also.

In general, grain size influences the yield strength of material in the way described by the Hall-Petch relation. That means yield stress increases as grain size becomes finer. But in this work, for the specimens undergone a large plastic deformation, the dislocation density is very high in the domain which under the nanocrystalized surface. So, not only the grain boundary but also the dislocation density determines the hardness of the material. It is also interesting that the process time has little effect on the hardness when the time exceeds 5 minutes. After processing about 5 minutes, it seems that the dislocation density tends to be saturated, so it does not increase with the process time. There exist two competitive processes: one is that the dislocation density increased with the plastic deformation; the other is that the movability of dislocation increased with the temperature of specimens, and the material might be recovered or even 


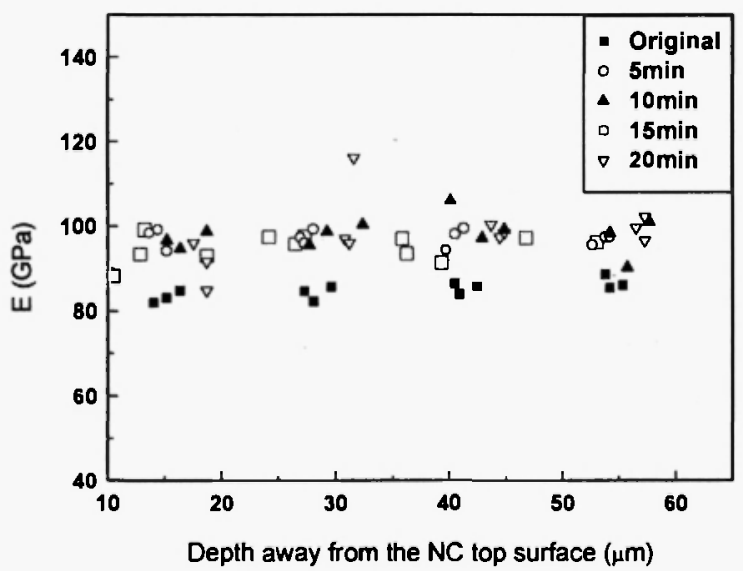

(a)

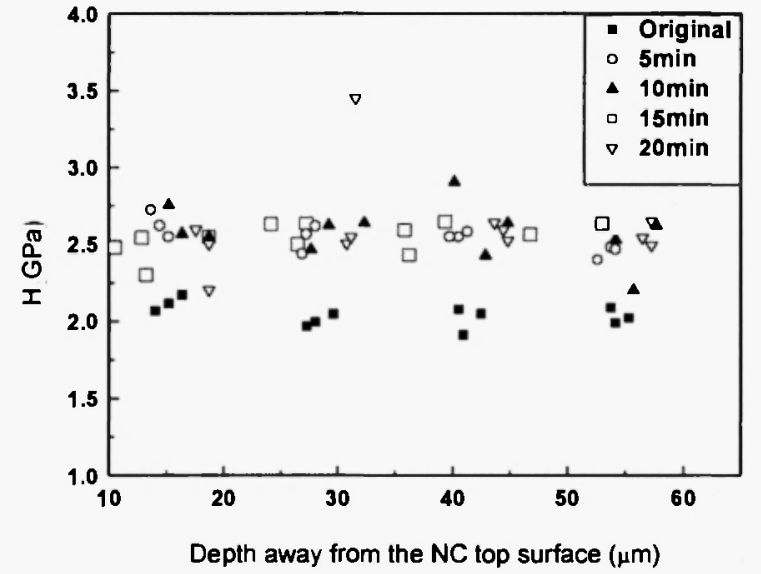

(b)

Fig.6 Elastic modulus(a) and nanohardness(b) versus distance from the nanocrystallized surface.

recrystallized. When process time beyond 5 minutes, with the shot peening and the increase of the temperature, a dynamic equilibrium of this two processes is reached.

It is very interesting that the elastic modulus also increased by $8 \%$ compared to the original state of material. It cannot be explained by dislocation theory simply, and needs further study.

\section{Concluding Remarks}

This paper presents the variations of hardness and elastic modulus for an aluminum alloy before and after nanocrystallization induced by HSSP through nano-indentation experiments. The relationship between hardness/elastic modulus and the grain size is investigated combined with transmission electron microscopy. The results of nanoindentation experiment show that the elastic modulus and the hardness of nanocrystal layers increase by $8 \%$ and $20 \%$, respectively. The variation of elastic modulus and hardness was little with the distance from nanocrystalized surface within the thin deformed layer and the process time.

\section{References}

[1] Gleiter, H. Nanostructured materials: basic concepts and microstructure, Acta Materialia, 48 (1) $(2000) 1-29$

[2] Valiev, R.Z., Islamgaliev, R.K. and Alexandrov, I.V. Progress in Materials Science, 45(2) (2000) 103-189

[3] Deng, Z.M., Hong, Y.S. and Zhu, C. SPD Nanostructured Materials Techniques and Mechanical Properties, National Conference on mechanical and west development, 2001, Wulumuqi

[4] Liu, G., Wang, S.C., Lou, X.F., Lu, J. and Lu, K. Low carbon steel with nanostructured surface layer induced by high-energy shot peening, Scripta Materialia, 44(8-9) (2001) 1791-1795 1359-6462

[5] Wu, X., Tao, N., Hong, Y., Xu, B., Lu, J. and Lu, K. Microstructure and evolution of mechanically -induced ultrafine grain in surface layer of $\mathrm{Al}$ alloy subjected to USSP, Acta Materialia, 50 (8) (2002) 2075-2084

[6] Ma, Y.L. Master Dissertation of Institute of Mechanics, Chinese Academy of Sciences, 2001

[7] Wu, X., Hong, Y., Lu, J. and Lu, K. Fabrication and nanostructured surface layers of $\mathrm{Al}$-alloys by surface mechanical attrition. MRS Proceedings, 697 (2002) p.8.142-8. 146 